\title{
Hernia Repair During Endoscopic Extraperitoneal Radical Prostatectomy: Outcome After 93 Cases
}

\author{
Minh Do, M.D., Evangelos N. Liatsikos, M.D., Ph.D., ${ }^{1,2}$ Panagiotis Kallidonis, M.D., ${ }^{2}$ \\ Andrew W. Wedderburn, M.D., ${ }^{3}$ Anja Dietel, M.D., \\ Kevin J. Turner, M.D., ${ }^{3}$ and Jens-Uwe Stolzenburg, M.D., Ph.D.
}

\begin{abstract}
Objective: To investigate the outcome of preperitoneal inguinal hernia mesh repairs performed during endoscopic extraperitoneal radical prostatectomy (EERPE).

Patients and Methods: Ninety-three patients underwent inguinal hernia repair during 2125 EERPEs performed between 2002 and 2008. Seventy-seven patients had a unilateral hernia and 16 bilateral inguinal hernias. Patients were treated with EERPE or nerve-sparing EERPE and pelvic lymphadenectomy (if indicated) for localized prostate cancer.

Results: The mean age of the patients was 63 years (range 49-75 years). Operative time was 150 minutes (range 85-285 minutes) and estimated mean blood loss was $240 \mathrm{~mL}$ (range 30-600 mL). Blood transfusion was never deemed necessary. No conversions to open surgery took place. The mean duration of catheterization was 6.5 days (range 4-25 days). One patient developed a pelvic haematoma, three patients had symptomatic pelvic lymphoceles, and one developed an anastomotic stricture. One patient suffered a rectal injury during the procedure and another developed deep venous thrombosis. The only complication of hernia repair was mild penile bruising and edema. During the follow-up period, we have never observed mesh infection or hernia recurrence.

Conclusion: EERPE combined with either a unilateral or bilateral laparoscopic hernia repair appears to be a safe and effective procedure. The incidence of complications related to either EERPE or the hernia repair was not increased. Oncological and functional outcome of EERPE seems not to be influenced by the performance of inguinal hernia repair.
\end{abstract}

\section{Introduction}

L APAROSCOPIC OR ENDOSCOPIC extraperitoneal radical prostatectomy (EERPE) has been described as a safe and effective procedure for the treatment of localized prostate cancer. ${ }^{1-5}$ The incidence of prostate cancer is increasing and both recognized and unrecognized inguinal hernias are common in the age group of men suffering from prostate cancer. Therefore, these two conditions commonly coexist.

Several methods of inguinal hernia repair are available, both open and laparoscopic. There are two well-described methods of laparoscopic repair of inguinal hernia: the totally extraperitoneal patch (TEP) and the transabdominal preperitoneal patch (TAPP). Regardless of the approach, laparoscopic repair of inguinal hernias is a well-established technique due to its safety, efficacy, and patient satisfaction. Recurrence rates were reduced below $5 \%$ when mesh implantation was introduced.,
TEP is associated with better results (recurrence rates, postoperative pain, and seroma formation) than the transabdominal preperitoneal patch, whereas the former technique provides access to the contralateral side without the need of further incisions. ${ }^{6}$ The TEP approach has been associated with efficacy in the management of inguinal hernia, whereas longterm follow-up demonstrates excellent functional results and a low recurrence rate. ${ }^{8}$ The hernia repair can be performed after a lymph node dissection if the latter procedure is also indicated during the EERPE.

The extraperitoneal technique (EERPE) has been established as a method for the management of localized prostate cancer and possesses several advantages over the transperitoneal method of laparoscopic radical prostatectomy, which was originally described by Guillonneau. ${ }^{9,10}$ Both techniques can be combined with concomitant inguinal hernia repair, but the extraperitoneal approach avoids any contact of the mesh

\footnotetext{
${ }^{1}$ Department of Urology, University of Leipzig, Leipzig, Germany.

${ }^{2}$ Department of Urology, University of Patras Medical School, Patras, Greece.

${ }^{3}$ Department of Urology, Royal Bournemouth Hospital, Bournemouth, United Kingdom.
} 

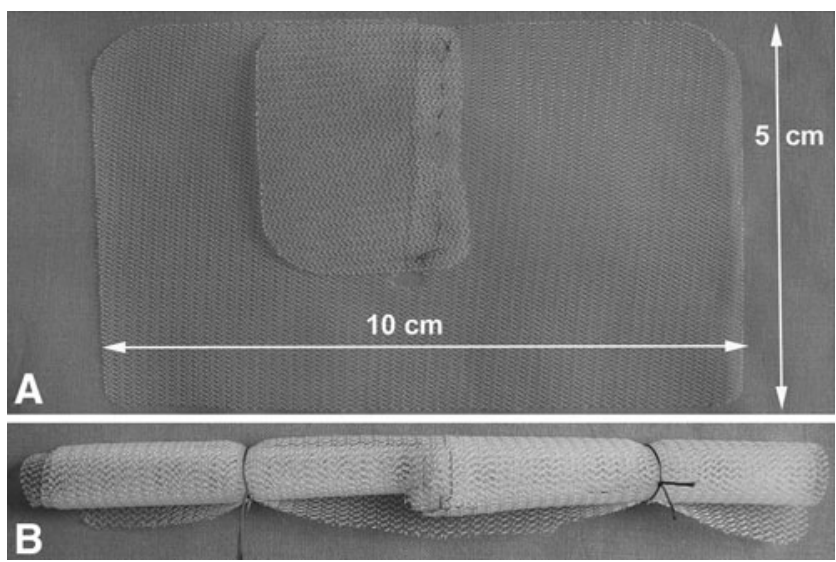

FIG. 1. (A) The modified mesh including the mesh flap. (B) The mesh rolled and tied by sutures, ready to be inserted.

with bowel markedly reducing the risk of subsequent adhesions. We currently present our experience with the performance of TEP inguinal hernia repair along with EEPRE.

\section{Patients and Methods}

Between December 2001 and November 2008, we performed EERPE on 2125 consecutive patients with clinically localized prostate cancer (cT1-cT2c). Ninety-three patients concomitantly underwent TEP hernia repair. The technique of EERPE has been previously described in detail. ${ }^{4,10}$ The EERPE is the standard of surgical management of localized cancer at our institutions. Bilateral nerve sparing EERPE (nsEERPE) is performed in preoperatively potent patients with $\mathrm{T} 1$ or T2 disease, prostate-specific antigen $<10 \mathrm{mg} / \mathrm{mL}$, and Gleason score $\leq 3+4$. Pelvic lymph node dissection is performed according to the following indications: prostate-specific antigen $>10 \mathrm{ng} / \mathrm{mL}$ and/or Gleason sum $>6$ or G3 tumor. Reducible inguinal hernias regardless of their size were managed in the current series. Irreducible hernias were not attempted.

The preoperative preparation of patients did not require any deviation to the standard preparation for the remaining EERPE procedures performed at our institutions. In addition, the combined performance of a TEP hernia repair with
EERPE does not require significant modification of the standard EERPE (or nsEERPE) technique. The hernia is mobilized before the prostatectomy and lymph node dissection (if indicated). Nevertheless, the actual hernia repair is performed after completion of the anastomosis. Indirect inguinal hernias require the sac to be retracted and dissected free from the cord. Traction and counter traction are used, but the dissection is occasionally almost completely accomplished during balloon inflation and preparation of the preperitoneal space.

A $10-\mathrm{cm}-\mathrm{by}-5-\mathrm{cm}$ Premilene ${ }^{\circledR}$ (B:Braun) mesh is prepared by making a $6-\mathrm{cm}$ cut vertically $7.5 \mathrm{~cm}$ from the cephalic end. A small $0.5-\mathrm{cm}$ hole is prepared for the cord structures. The cut is covered by a further $6.5-\mathrm{cm}-\mathrm{by}-4-\mathrm{cm}$ sheet of mesh that is sutured into place using prolene sutures. This acts as a flap over the cut (Fig. 1A). The flap is temporarily held back leaving the cut mesh free to be placed around the spermatic cord. The mesh is rolled up and tied with two stay sutures to allow easy placement via the $12-\mathrm{mm}$ trocar (Fig. 1B). Once inside the stay sutures are cut and the mesh unfolded (Fig. 2A, B). It is positioned in place around the spermatic cord from the pubic symphysis in the midline to the anterior iliac spine laterally (Fig. 3A, B). No sutures or clips are used to hold the mesh in place. Moreover, it is possible for clips to migrate after the procedure. As the $\mathrm{CO}_{2}$ is released, the abdominal pressure holds the mesh in place.

Postoperative complications and functional results were recorded at 3, 6, and 12 months after EERPE. Patients not reporting the use of any pad were defined as continent. The use of 1 to 2 pads daily and normal physical activity (such as walking) was defined as mild incontinence. The use of $>2$ pads per day was considered as incontinence. Potency for the current patient series was evaluated by the use of International Index of Erectile Function-5 (IIEF-5) and Sexual Encounter Profile (SEP) diaries. A positive answer of the patient to the following SEP diary questions defined the patient as potent: SEP2 "Were you able to insert your penis into your partner's vagina?"; SEP3 "Did your erections last long enough to have sexual intercourse?"; and SEP5 "Were you satisfied with this sexual experience?"

A cystogram on the 5th postoperative day is performed in all patients who underwent EERPE. If no extravasation is observed, the catheter is removed.
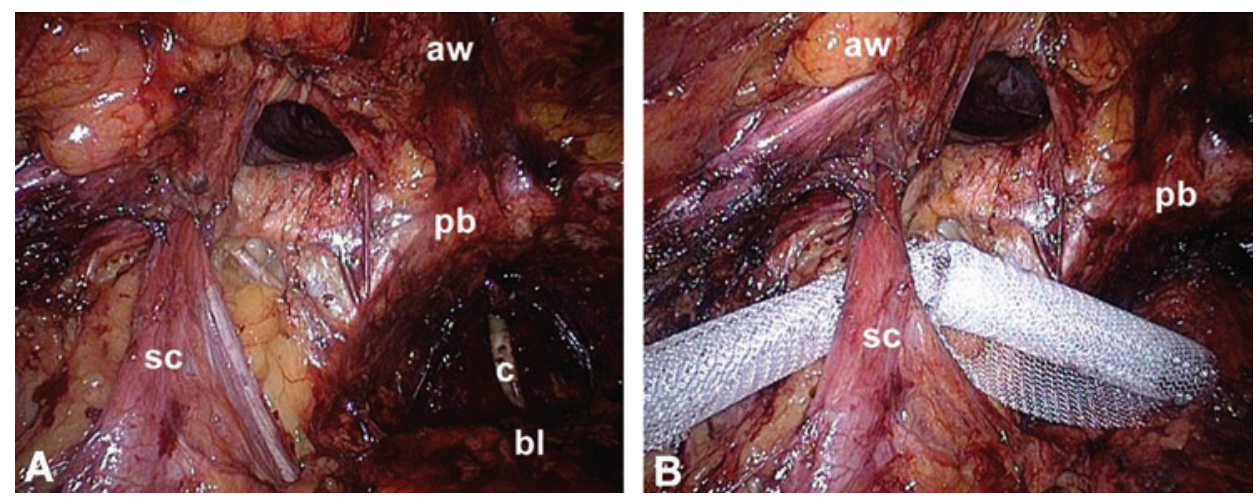

FIG. 2. (A) An inguinal hernia prepared for mesh placement. Anatomical landmarks are presented. (B) The mesh has been placed behind the spermatic cord and is ready to be unrolled. $\mathrm{aw}=$ abdominal wall; $\mathrm{pb}=$ pubic bone; $\mathrm{c}=$ catheter; $\mathrm{bl}=$ bladder; $\mathrm{sc}=$ spermatic cord . 


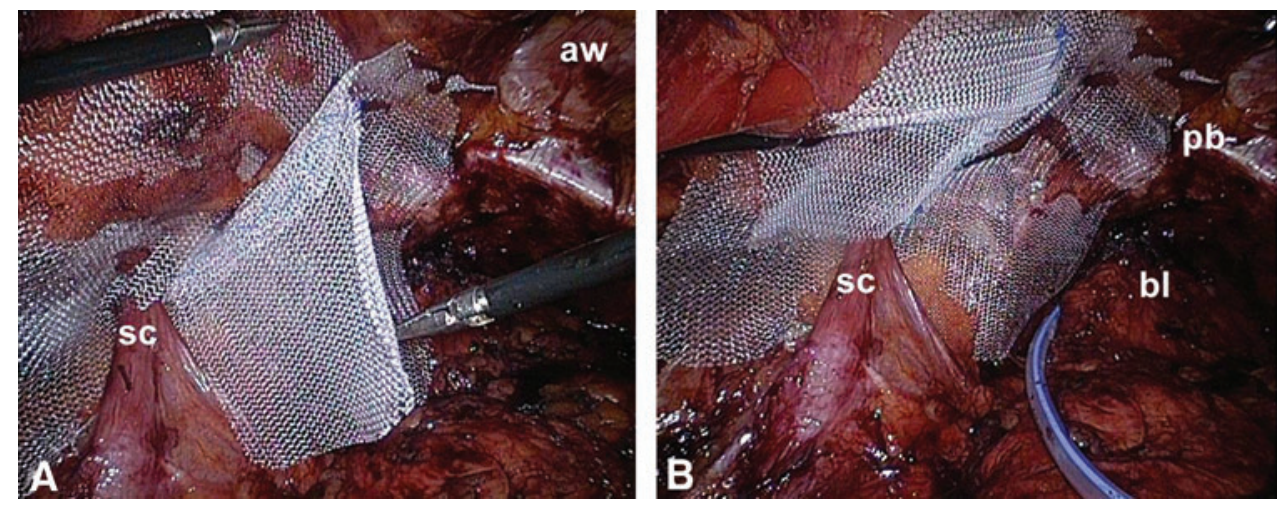

FIG. 3. (A) The mesh is placed in position. Notice the absence of anchoring sutures and clips. (B) The drainage is also in place and the removal of the gas is about to take place.

\section{Results}

The demographic data of 93 patients who underwent simultaneous TEP hernia repair with EERPE are presented in Table 1. Seventy-seven had a unilateral hernia and 16 bilateral hernias. The mean age of the patients was 63 years (range 49-75 years). Operative time was 150 minutes (range 85-285 minutes) and estimated mean blood loss was $240 \mathrm{~mL}$ (range 30-600 mL). Blood transfusion was never deemed necessary. No conversions to open surgery took place. Forty-eight out of the 93 patients underwent concomitant pelvic lymph node dissection. Thirty-four patients (36\%) underwent bilateral nsEERPE. Pathological results, continence, and potency outcome are summarized in Tables 2 and 3, respectively. The mean duration of catheterization was 6.5 days (range $4-25$ days).

One patient developed pelvic hematoma, which did not require intervention. Three patients had symptomatic pelvic lymphoceles, which required percutaneous drainage and one developed an anastomotic stricture requiring incision and dilatation intervention (Table 4). One patient suffered a rectal injury during the procedure, the hernia repair was completed after repair of the injury, and no further complications were observed. The most common complication was mild penile bruising and edema. During the follow-up period, we have never observed mesh infection or hernia recurrence.

\section{Discussion}

The current study reports probably the largest series of combined inguinal hernia repair and EERPE or laparoscopic

Table 1. Demographic Data

\begin{tabular}{lc}
\hline Number of cases & $93(16$ bilateral $)$ \\
Age & $63.5(49-75)$ \\
PSA & $9.3 \mathrm{ng} / \mathrm{mL}(1.73-34)$ \\
Operative time & 150 minutes $(85-285)$ \\
Blood loss & $240 \mathrm{~mL}(30-600)$ \\
Transfusion rate & $0 \%$ \\
Prostate size & $50.4 \mathrm{~g}(18-208)$ \\
Lymphadenectomy performed & $48(52 \%)$ \\
Bilateral intrafascial nerve sparing & $34(36 \%)$ \\
performed & \\
Duration of catheterisation & 6.5 days $(4-25)$ \\
\hline
\end{tabular}

PSA $=$ prostate-specific antigen. radical prostatectomy. As inguinal hernias are found in 5\%$10 \%$ of men, it will therefore be a common finding in men presenting with early prostate cancer. The incidence of inguinal hernias is reported to be higher in men who have undergone an open radical prostatectomy. ${ }^{11}$ Open radical prostatectomy after laparoscopic mesh inguinal hernia repair is technically difficult and may need to be abandoned. ${ }^{12,13}$ Nevertheless, laparoscopic radical prostatectomy is feasible in the above cases and should be considered. ${ }^{14,15}$

Open extraperitonal radical retropubic prostatectomy could be combined with inguinal hernia repair without additional complications. ${ }^{16,17}$ Lee et $\mathrm{al}^{18}$ reported repair of 48 hernias in 40 patients undergoing laparoscopic radical prostatectomy. They reported no increase in complications and no hernia recurrences. Two of their patients had lymphoceles that required drainage, but neither of these developed mesh infection, which is a possible complication of this combined procedure.

Finley et $\mathrm{al}^{19}$ reported inguinal hernia repair during robotassisted radical prostatectomy. Forty-nine repairs were performed in 40 patients. Five patients had previously undergone ipsilateral repair and three had bilateral repairs. Only 15 of the 40 patients were identified as having an inguinal hernia preoperatively. They reported an increase in operative time by 10 minutes to repair the hernia, had 15-month followup, and report no complications. They initially used absorbable mesh due to concerns regarding infection but found an

Table 2. Pathological Data

\begin{tabular}{cc}
\hline Staging & \\
pT2a & $11(11.8 \%)$ \\
pT2b & $2(2.2 \%)$ \\
pT2c & $47(50.4 \%)$ \\
pT3a & $22(23.7 \%)$ \\
pT3b & $10(10.8 \%)$ \\
pT4 & $1(1.1 \%)$ \\
Positive margins & \\
Overall & $14 / 93(15.1 \%)$ \\
pT2 & $3 / 60(5 \%)$ \\
pT3 & $10 / 32(31.3 \%)$ \\
pT4 & $1 / 1(100 \%)$ \\
PSA & \\
PSA $\leq 0.1 \mathrm{ng} / \mathrm{mL}$ at 6 months & $96.8 \%$ \\
PSA $\leq 0.1 \mathrm{ng} / \mathrm{mL}$ at 12 months & $95.7 \%$ \\
\hline
\end{tabular}


Table 3. Functional Results

\begin{tabular}{lc}
\hline Continence & \\
3-month continence & $74.7 \%$ \\
0 pad & $19.3 \%$ \\
1-2 pads & $6 \%$ \\
>2 pads & \\
6-month continence & $86.4 \%$ \\
0 pad & $12.3 \%$ \\
1-2 pads & $1.3 \%$ \\
>2 pads & \\
12-month continence $(n=75)$ & $94.7 \%$ \\
0 pad & $5.3 \%$ \\
1-2 pads & $0 \%$ \\
>2 pads & \\
Potency & $52 \%$ \\
6-month potency & $75 \%$ \\
12-month potency $(n=75)$ &
\end{tabular}

${ }^{a}$ Erections adequate for intercourse with or without PDE5 inhibitors.

PDE5 = phosphodiesterase-5.

unacceptable recurrence rate and therefore changed to a nonabsorbable mesh.

The current series demonstrates the feasibility and low morbidity of the hernia repair procedure in combination with EERPE. A major advantage of this combined procedure is the ease of performing the hernia repair. Standard technique for EERPE is used and the hernia repair is performed for hernias recognized preoperatively or found during the EERPE resulting in average operative time (150 minutes) similar to the total EERPE experience (150.7 minutes). ${ }^{10}$ Additional advantage is the mesh placement method that does not require suturing or clipping, as the mesh is secured in place when the extraperitoneal space regains its original volume with $\mathrm{CO}_{2}$ removal. Thus, the risk of clip migration is avoided. In addition, the extraperitoneal approach eliminates any contact to the bowel and prevents the risk of adhesions formation. Moreover, postoperative pain is reduced. ${ }^{6}$

The performance of laparoscopic hernia repair alone has a very low incidence of infection and recurrence. ${ }^{20}$ The latter observation was confirmed by the current series despite the concomitant performance of an oncological procedure. The incidence of complications related to EERPE such as symptomatic lymphoceles or bladder neck stenosis seems not to be influenced by the inguinal hernia repair. ${ }^{10}$

The short-term oncological outcome of EERPE remains similar to published EERPE series. ${ }^{10}$ Any changes to longer oncological results should not be expected as the hernia repair procedure does not interfere with EERPE and pelvic lym-

TABle 4. Complications

\begin{tabular}{ll} 
Perioperative & \\
$\quad$ Rectal injury & $1(1.1 \%)$ \\
Early postoperative (<1 month postoperatively) & \\
$\quad$ Anastamotic leak & $5(5.4 \%)$ \\
Bleeding/hematoma & $1(1.1 \%)$ \\
Urinary tract infection & $2(2.2 \%)$ \\
Symptomatic lymphocele & $2(3.3 \%)$ \\
$\quad$ Deep venous thrombosis & $1(1.1 \%)$ \\
Late complications ( $>1$ month postoperatively) & \\
$\quad$ Anastamotic stricture & $1(1.1 \%)$ \\
\hline
\end{tabular}

phadenectomy (if indicated). The hernia repair is performed after the completion of the prostatectomy (and lymphadenectomy).

The functional outcome of current series is also similar to total EERPE experience. ${ }^{10} \mathrm{Up}$ to $94.5 \%$ of the patients were continent, whereas $75 \%$ of patients undergoing either a unilateral or bilateral nsEERPE reported adequate erections for intercourse at 12 months. The functional outcome of EERPE remains unchanged with concomitant performance of the procedure.

Limitation of the current report is the retrospective nature of the study. Although the data were prospectively recorded in an extensive database at our institutions, these data were retrospectively collected and evaluated. The presented data represent the experience of specialized centers in laparoscopic urological surgery with significant experience in the field.

\section{Conclusion}

EERPE combined with either a unilateral or bilateral hernia repair appears to be a safe and effective procedure. The incidence of complications related to either EERPE or the hernia repair was not increased. Oncological and functional outcome of EERPE seems not to be influenced by the performance of inguinal hernia repair.

\section{Disclosure Statement}

No competing financial interests exist.

\section{References}

1. Guillonneau B, el-Fettouh $\mathrm{H}$, Baumert $\mathrm{H}$, et al. Laparoscopic radical prostatectomy: Oncological evaluation after 1,000 cases a Montsouris Institute. J Urol 2003;169:1261-1266.

2. Eden CG, Neill MG, Louie-Johnsun MW. The first 1000 cases of laparoscopic radical prostatectomy in the UK: Evidence of multiple "learning curves". BJU Int 2009;103:1224-1230.

3. Frota R, Turna B, Barros R, Gill IS. Comparison of radical prostatectomy techniques: Open, laparoscopic and robotic assisted. Int Braz J Urol 2008;34:259-268; discussion 68-69.

4. Stolzenburg JU, Rabenalt R, Do M, et al. Intrafascial nervesparing endoscopic extraperitoneal radical prostatectomy. Eur Urol 2008;53:931-940.

5. Ficcara V, Novara G, Artibani W, et al. Retropubic, laparoscopic and robot-assisted radical prostatectomy: A systematic review and cumulative analysis of comparative studies. Eur Urol 2009;55:1037-1063.

6. Abrazhda D, Hübner M, Foletti A, Demartines N, Vuilleumier H. [Pain after inguinal hernia repair: what to do?]. Rev Med Suisse 2010;6:1288-1291.

7. Messaris E, Nicastri G, Dudrick SJ. Total extraperitoneal laparoscopic inguinal hernia repair without mesh fixation: Prospective study with 1-year follow-up results. Arch Surg 2010;145:334-338.

8. Kouhia ST, Huttunen R, Silvasti SO, et al. Lichtenstein hernioplasty versus totally extraperitoneal laparoscopic hernioplasty in treatment of recurrent inguinal hernia-a prospective randomized trial. Ann Surg 2009;249:384-387.

9. Guillonneau B, Vallancien G. Laparoscopic radical prostatectomy: The Montsouris technique. J Urol 2000;163: 1643-1649.

10. Stolzenburg JU, Kallidonis P, Do M, et al. Endoscopic extraperitoneal radical prostatectomy: Evolution of the tech- 
nique and experience with 2400 cases. J Endourol 2009;23: 1467-1472.

11. Ichioka K, Yoshimura K, Utsunomiya N, et al. High incidence of inguinal hernia after radical retropubic prostatectomy. Urology 2004;63:278-281.

12. Cook H, Afzal N, Cornaby AJ. Laparoscopic hernia repairs may make subsequent radical retropubic prostatectomy more hazardous. BJU Int 2003;91:729.

13. Katz EE, Patel RV, Sokoloff MH, Vargish T, Brendler CB. Bilateral laparoscopic inguinal hernia repair can complicate subsequent radical retropubic prostatectomy. J Urol 2002; 167(2 Pt 1):637-638.

14. Erdogru T, Teber D, Frede T, Marrero R, Hammady A, Rassweiler J. The effect of previous transperitoneal laparoscopic inguinal herniorrhaphy on transperitoneal laparoscopic radical prostatectomy. J Urol 2005;173: 769-772.

15. Stolzenburg JU, Anderson C, Rabenalt R, Do M, Ho K, Truss MC. Endoscopic extraperitoneal radical prostatectomy in patients with prostate cancer and previous laparoscopic inguinal mesh placement for hernia repair. World J Urol 2005; 23:295-299.

16. Schlegel PN, Walsh PC. The use of the preperitoneal approach for the simultaneous repair of inguinal hernia during surgery on the bladder and prostate. World J Surg 1989;13: 555-559.

17. Manoharan M, Gomez P, Soloway MS. Concurrent radical retropubic prostatectomy and inguinal hernia repair through a modified Pfannenstiel incision. BJU Int 2004;93: 1203-1206.
18. Lee BC, Rodin DM, Shah KK, Dahl DM. Laparoscopic inguinal hernia repair during laparoscopic radical prostatectomy. BJU Int 2007;99:637-639.

19. Finley DS, Rodriguez E, Jr., Ahlering TE. Combined inguinal hernia repair with prosthetic mesh during transperitoneal robot assisted laparoscopic radical prostatectomy: A 4-year experience. J Urol 2007;178(4 Pt 1):1296-1299; discussion 9300.

20. Moon V, Chaudry GA, Choy C, Ferzli GS. Mesh infection in the era of laparoscopy. J Laparoendosc Adv Surg Tech A 2004;14:349-352.

Address correspondence to: Evangelos N. Liatsikos, M.D., Ph.D.

Department of Urology University of Patras Medical School

Rion

Patras 26500

Greece

E-mail: liatsikos@yahoo.com

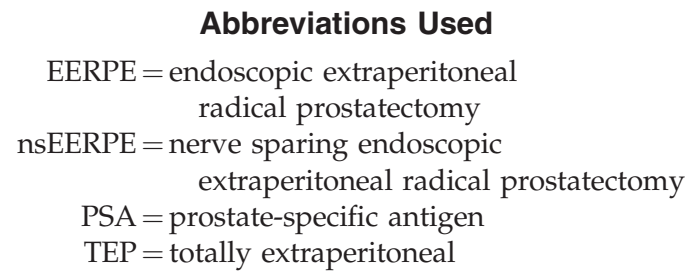


